\title{
Gradual Tolerance of Metabolic Activity Is Produced in Mesolimbic Regions by Chronic Cocaine Treatment, while Subsequent Cocaine Challenge Activates Extrapyramidal Regions of Rat Brain
}

\author{
R. P. Hammer, Jr. and E. S. Cooke \\ Laboratory of Cellular and Molecular Neuropharmacology, University of Hawaii School of Medicine, Honolulu, Hawaii \\ 96822
}

Acute administration of cocaine is known to enhance extracellular dopamine levels in the striatum and to activate immediate-early gene expression in striatal neurons. Regional cerebral metabolic rate for glucose $\left(\mathrm{rCMR}_{\mathrm{glc}}\right)$ reportedly increases in extrapyramidal and mesolimbic brain regions in response to acute cocaine treatment. However, chronic administration attenuates the cocaine-induced enhancement of regional dopamine response and the induction of immediate-early gene expression in these regions. Chronic treatment also produces tolerance to cocaine's reinforcing effects. Thus, differential responses to cocaine occur with increasing length of treatment. Therefore, we examined the time course of effects of repeated daily cocaine treatment on $\mathrm{rCMR}_{\mathrm{glc}}$ in rat brain. Acute administration of $10 \mathrm{mg} / \mathrm{kg}$ cocaine slightly increased rCMR $_{\mathrm{glc}}$ in mesolimbic and extrapyramidal regions. However, no significant effects were observed until more than $7 \mathrm{~d}$ of treatment, whereupon $\mathbf{r C M R}_{\text {grc }}$ was reduced compared to saline treatment in the infralimbic portion of the medial prefrontal cortex, nucleus accumbens, olfactory tubercle, habenula, amygdala, and a few other brain regions. In contrast, after $13 \mathrm{~d}$ of $10 \mathrm{mg} / \mathrm{kg}$ cocaine treatment, challenge with $30 \mathrm{mg} / \mathrm{kg}$ cocaine increased $\mathrm{rCMR}_{\mathrm{glc}}$ in the striatum, globus pallidus, entopeduncular nucleus, subthalamus, substantia nigra pars reticulata, and a few other regions without affecting limbic or mesolimbic regions. Thus, repeated daily treatment with a low dose of cocaine gradually decreased metabolic activity particularly in mesolimbic regions. Subsequent treatment with a higher dose produced metabolic activation mostly in extrapyramidal regions: This effect of chronic treatment could represent tolerance to the initial metabolic response, which can be replicated thereafter but only by increasing the drug dose. These results suggest that tolerance to the metabolic effects of cocaine in selective mesolimbic circuits may contribute to the development of behavioral dependence with repeated exposure.

\footnotetext{
Received Sept. 23, 1993; revised Dec. 27, 1993; accepted Jan. 13, 1994.

This work was supported by USPHS Awards DA06645 and Research Career Development Award NS01161 to R.P.H. We thank Anna R. Mateo and Blanche B. Young for assistance in various parts of these studies, and Wendy S. Pires for expert technical support.

Correspondence should be addressed to Dr. Ronald P. Hammer, Department of Anatomy and Reproductive Biology, University of Hawaii School of Medicine, 1960 East-West Road, Honolulu, HI 96822

Copyright (C) 1994 Society for Neuroscience $0270-6474 / 94 / 144289-10 \$ 05.00 / 0$
}

[Key words: chronic cocaine, 2-deoxyglucose, regional cerebral metabolic rate, psychostimulant activation, tolerance, dependencel

Chronic cocaine exposure produces various behavioral, neurochemical, and pharmacological alterations in rodent brain. Repeated administration produces sensitization of locomotor response to subsequent cocaine treatment (Kalivas et al., 1988; Weiss et al., 1989), which is related to the enhancement of extracellular dopamine levels in the nucleus accumbens (NAc) produced by cocaine (Kalivas et al., 1988; Hurd et al., 1989; Pettit and Justice, 1989). However, chronic treatment attenuates NAc dopamine response compared to that produced by acute cocaine treatment (Hurd et al., 1989), even in the presence of locomotor sensitization (Segal and Kuczenski, 1992; Kalivas and Duffy, 1993). Furthermore, immediate-early gene induction in NAc neurons is desensitized by chronic cocaine treatment compared to acute treatment (Hope et al., 1992). Thus, certain biochemical parameters decrease following chronic cocaine exposure.

Acute cocaine treatment increases metabolic activity in various extrapyramidal (London et al., 1986; London, 1990) and mesolimbic (Porrino et al., 1988; Kornetsky et al., 1991) regions of rat brain, which is likcly duc to cocainc-induced alteration of dopaminergic activity (McCulloch et al., 1982; Trugman and James, 1993). In contrast, regional metabolism is reduced during withdrawal following chronic cocaine administration (Clow and Hammer, 1991; Hammer et al., 1993). In addition, recent positron emission tomographic studies of cerebral metabolism (London et al., 1990) and blood flow (Pearlson et al., 1993) in human cocaine abusers demonstrate regional reductions following cocaine administration. Thus, it is possible that cocaine treatment produces different effects in chronically treated animals from those observed in naive animals (as used in acute drug trials). Therefore, we examined the time course of regional metabolic response following repeated daily cocaine treatment using the quantitative ${ }^{14} \mathrm{C}$-2-deoxyglucose (2DG) autoradiographic procedure (Sokoloff et al., 1977).

Recent studies have shown that chronic treatment with or self-administration of cocaine increased the frequency of subsequent cocaine self-administration (Emmett-Oglesby et al., 1993) as well as the dose required to support self-administration (Emmett-Oglesby and Lane, 1992), suggesting the development of tolerance to the reinforcing efficacy of the drug. If regional metabolic response following chronic cocaine treatment does differ from the response in naive animals due to the develop- 
ment of tolerance, then a higher dose might activate the affected brain circuits, thereby replicating the initial metabolic response. Therefore, the effect of challenge with a high cocaine dose on regional metabolic response was examined in chronically treated rats.

The results demonstrate that chronic cocaine treatment reduces regional metabolic response primarily in mesolimbic regions of the rodent brain. Furthermore, subsequent challenge with a higher dose of cocaine selectively activates extrapyramidal, but not mesolimbic regions. This suggests that cellular activity in specific circuits is reduced following chronic cocaine treatment. Acute effects can be reproduced thereafter, but only upon administration of a higher cocaine dose. Such effects on selective brain circuits might underlie the development of dependence in response to chronic cocaine exposure.

\section{Materials and Methods}

Subjects. Male Sprague-Dawley rats (Simonsen, Gilroy, CA), weighing $250-325 \mathrm{gm}$ at the time of treatment, were housed individually in suspended metal cages in light- (on from $0600-2000 \mathrm{hr}$ ) and temperature- $\left(23^{\circ} \mathrm{C}\right)$ controlled rooms with ad libitum access to food and water. Animals were allowed to acclimate to these conditions for at least $4 \mathrm{~d}$, after which they were randomly placed into one of six groups in experiment 1 , or one of three groups in experiment 2 . All studies were carried out in accord with the policies of the Guide for the Care and Use of Laboratory Animals (NIH Publication NIH860-23), and were approved by the University of Hawaii Institutional Animal Care and Use Committee.

Treatment paradigm in experiment 1 . Animals were weighed and treated daily for $14 \mathrm{~d}$ with an intraperitoneal injection $(1 \mathrm{ml} / \mathrm{kg})$ of either $0.9 \%$ saline vehicle or $10 \mathrm{mg} / \mathrm{kg}$ cocaine $\mathrm{HCl}$ administered between 1100 and $1200 \mathrm{hr}$. Animals received either daily saline injections or cocaine injections on the last $1,3,7,10$, or $14 \mathrm{~d}$ of treatment after initial daily injections of saline.

Treatment paradigm in experiment 2. Animals were weighed daily and received intraperitoneal injections $(1 \mathrm{ml} / \mathrm{kg})$ of either saline or cocaine for $14 \mathrm{~d}$. Cocaine treatment groups received $10 \mathrm{mg} / \mathrm{kg}$ on each of the first $13 \mathrm{~d}$ followed on the last day by either $10 \mathrm{mg} / \mathrm{kg}$ or $30 \mathrm{mg} / \mathrm{kg}$. Thus, the saline and $10 \mathrm{mg} / \mathrm{kg}$ daily cocaine groups replicated these treatment groups in experiment 1.

2-Deoxyglucose autoradiographic procedures. On the final day of the experiment, animals were anesthetized with halothane, and femoral arterial and venous cannulas were implanted and passed subcutaneously to exit through a small incision on the hack. During this procedure, the femoral nerve was carefully preserved to ensure competent motor performance in the affected limb. The 2DG procedure was then performed on freely moving rats as previously described (Crane and Porrino, 1989). Animals were allowed to recover for at least $4 \mathrm{hr}$, after which the final dose of cocaine or saline was administered. The rats were then placed into a novel environment (shoebox cage lined with benchtop paper), and arterial samples were taken to determine hematocrit and baseline plasma glucose values. Ten minutes after drug treatment, a pulse of ${ }^{14} \mathrm{C}$ 2DG (125 $\mu \mathrm{Ci} / \mathrm{kg}$; American Radiolabeled Chemicals, St. Louis, MO) was injected intravenously and timed arterial samples were collected during a $45 \mathrm{~min}$ period. Blood pressure was monitored $5 \mathrm{~min}$ after 2DG injection. Locomotor behavior was observed and qualitatively assessed during this period, although no quantitative measurements were accomplished. Animals were then given a lethal intravenous injection of $65 \mathrm{mg} / \mathrm{kg}$ sodium pentobarbital, and brains were rapidly removed and frozen in $-30^{\circ} \mathrm{C} 2$-methylbutane. Blood samples were centrifuged and plasma glucose and deoxyglucose values were determined using a glucose analyzer and liquid scintillation spectroscopy, respectively. Brains were sectioned in a $-17^{\circ} \mathrm{C}$ cryostat, and sections were mounted on coverslips that were dried on a $60^{\circ} \mathrm{C}$ hot plate. Coverslips were mounted on cardboard and apposed to x-ray film (EMC-1, Kodak) together with calibrated acrylic ${ }^{14} \mathrm{C}$ radiostandards (American Radiolabeled Chemicals, St. Louis, MO). Films were developed 12 d later using GBX developer and fixer (Kodak). Autoradiographs were utilized only if optimal labeling (e.g., no visible diffusion of 2DG label and good contrast for regional identification) was present.

The resulting autoradiographs were analyzed using specialized image analysis software (IMAGE-mg; Wayne Rasband, Gunter Mies; NIMH, Bethesda, MD) on a Macintosh IIfx computer with a frame grabber board (Data Translation, Marlboro, MA) interfaced to a Sony XC-77 CCD camera on a DC-stabilized light table. Regional identification was accomplished when required by superimposing the image of the Nisslstained section upon its corresponding autoradiograph. Calibrated regional optical density values were measured in 61 individual regions in experiment 1 , and 66 regions in experiment 2 . Regional cerebral metabolic rate for glucose $\left(\mathrm{rCMR}_{\mathrm{glc}}\right)$ was calculated using the measured values for both plasma ${ }^{19} \mathrm{C}-2 \mathrm{DG}$ and glucose concentration, the published constants derived using the operational equation of Sokoloff (Sokoloff et al., 1977), and the calibrated regional optical density values.

$\mathrm{rCMR}_{\mathrm{glc}}$ results from experiment 1 were compared across treatment groups using one-way analysis of variance to examine the main effect of length of cocaine treatment followed by Newman-Keuls post hoc comparisons where appropriate to determine the loci of the effect. In experiment 2 , treatment groups were compared using Duncan's multiple range test. The results were determined to be significantly different if $p$ $\leq 0.05$ in all comparisons.

\section{Results}

\section{Length of cocaine treatment}

Acute cocaine treatment produced a slight but nonsignificant increase of $\mathrm{rCMR}_{\mathrm{glc}}$ in various regions, especially in structures of the mesolimbic forebrain (Table 1). Additional treatment produced little alteration from control values until 10 consecutive days of treatment (Fig. 1), after which $\mathrm{rCMR}_{\text {glc }}$ was significantly reduced compared to saline or acute cocaine treatment in the NAc, olfactory tubercle (OT), and piriform cortex (PC). rCMR $_{\text {glc }}$ was significantly reduced compared to acute cocaine treatment in the basolateral nucleus of the amygdala (ABL). After $14 \mathrm{~d}$ of cocaine treatment, $\mathrm{rCMR}_{\mathrm{glc}}$ was significantly reduced compared to saline or acute cocaine treatment in the NAc, $\mathrm{ABL}, \mathrm{PC}$, as well as the ventromedial caudatoputamen $(\mathrm{CP})$ and cingulate cortex. At this time point, $\mathrm{rCMR}_{\mathrm{glc}}$ in the OT was significantly reduced compared to acute cocaine treatment. Each of the above regions displayed significant main effects of length of cocaine treatment. No alterations were observed in white matter, as measured in the corpus callosum.

In the test environment, animals were observed to be more active following acute cocaine treatment than after any other treatment examined. Following $3 \mathrm{~d}$ or more of cocaine treatment, animals reacted similarly to those receiving saline treatment.

\section{Effect of repeated cocaine treatment and subsequent challenge with a higher dose}

Repeated treatment with $10 \mathrm{mg} / \mathrm{kg}$ cocaine for $14 \mathrm{~d}$, again, significantly reduced $\mathrm{rCMR}_{\text {gic }}$ compared to chronic saline treatment in NAc, OT, CP, PC, and amygdala, as well as in the infralimbic portion of the medial prefrontal cortex $\left(\mathrm{MPf}_{\mathrm{il}}\right)$, substantia nigra pars compacta ( $\mathrm{SNc}$ ), habenula, locus ceruleus (LC), and $a$ few other brain regions (Table 2 ). No significant alterations were observed in white matter.

When $30 \mathrm{mg} / \mathrm{kg}$ cocaine was administered on the final treatment day, rCMR $_{\text {glc }}$ remained significantly lower compared to chronic saline treatment in some brain regions, including the $\mathrm{MPf}_{\mathrm{il}}$, lateral habenula (LH), inferior colliculus, and superior olive. In contrast, $\mathrm{rCMR}_{\mathrm{glc}}$ increased significantly compared to intermittent treatment with $10 \mathrm{mg} / \mathrm{kg}$ cocaine in the $\mathrm{CP}$, globus pallidus (GP), entopeduncular nucleus (EP), subthalamus (STh), substantia nigra pars reticulata ( $\mathrm{SNr}$ ), somatosensory and motor cortices, periaqueductal gray matter (PAG), and LC. This effect was significantly different compared to chronic saline treatment only in the GP, STh, and SNr (Fig. 2). 

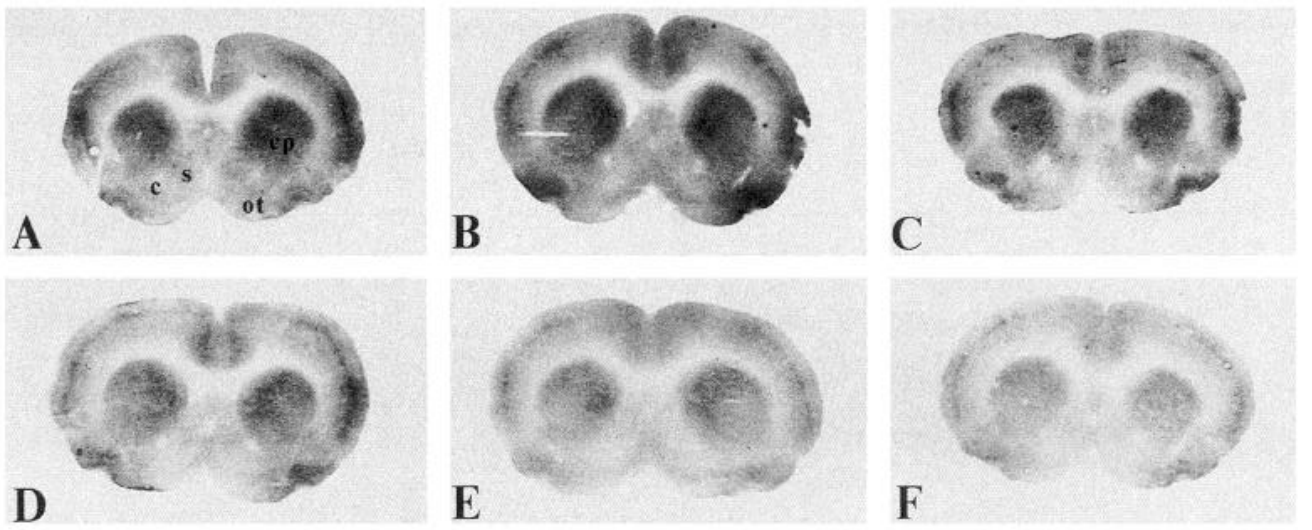

Figure 1. Regional metabolic effects of repeated daily $10 \mathrm{mg} / \mathrm{kg}$ cocaine treatment are dependent upon the length of treatment. Autoradiographs are from sections taken through the rostral portion of the nucleus accumbens (NA) at $1.2 \mathrm{~mm}$ anterior to bregma (Paxinos and Watson, 1986). $A$, Saline treatment for $14 \mathrm{~d}$. B. Acute cocaine treatment ( $1 \mathrm{~d}$ cocaine following $13 \mathrm{~d}$ saline treatment). $C$, Three days cocaine (following $11 \mathrm{~d}$ saline treatment). $D$, Seven days cocaine (following $7 \mathrm{~d}$ saline treatment). $E$, Ten days cocaine (following $4 \mathrm{~d}$ saline treatment). $F$, Fourteen days cocaine treatment. $\mathrm{rCMR}_{\mathrm{glc}}$ increases slightly with acute treatment $(B)$, returns to normal after 3 or $7 \mathrm{~d}$ treatment $(C$ and $D)$, and thereafter decreases significantly in both NA core $(c)$ and shell $(s)$, as well as in olfactory tubercle $(o t)$ and part of the caudatoputamen $(c p)$ after 10 and $14 \mathrm{~d}$ treatment $(E$ and $F)$.

Chronic treatment with $10 \mathrm{mg} / \mathrm{kg}$ cocaine produced similar locomotor behavior to that observed following saline treatment. In contrast, locomotor activity substantially increased after treatment with $30 \mathrm{mg} / \mathrm{kg}$ cocaine on the final treatment day.

\section{Discussion}

\section{Effects of chronic cocaine treatment}

Cocaine binds to catecholamine transporters, thereby blocking reuptake and increasing extracellular transmitter levels in terminal regions. Thus, acute cocaine treatment in naive rats dramatically elevates extracellular dopamine levels in the NAc (Hurd et al., 1989; Pettit and Justice, 1989). The reinforcing efficacy of cocaine is probably due to its effect on dopamine transporters (Ritz et al., 1987) in the NAc (Roberts et al., 1980). However, the effects of chronic treatment are different from those of acute treatment. For example, the extracellular dopamine response in the NAc is attenuated compared to that produced by acute cocaine exposure (Hurd et al., 1989), even in the presence of locomotor sensitization (Segal and Kuczenski, 1992; Kalivas and Duffy, 1993). Such attenuated dopamine response to cocaine after chronic exposure could represent a neurochemical manifestation of tolerance.

Chronic treatment also produced tolerance to the reinforcing efficacy of cocaine, whereby repeated exposure increased the rate of self-administration and the threshold dose required to support self-administration in rats (Emmett-Oglesby and Lane, 1992; Emmett-Oglesby et al., 1993). Continuous cocaine infusion produced the same effect in rhesus monkeys (Woolverton and Kleven, 1988); however, differential behavioral responses to continuous and intermittent cocaine treatment have been observed in rats (King et al., 1992) and mice (Reith et al., 1987). Such a behavioral demonstration of tolerance could result from a physiological condition of dependence.

Continuous cocaine treatment also produced tolerance to the

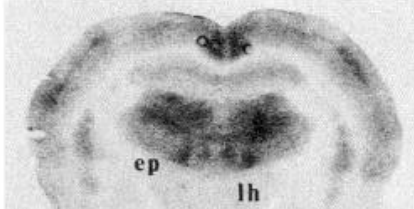

A

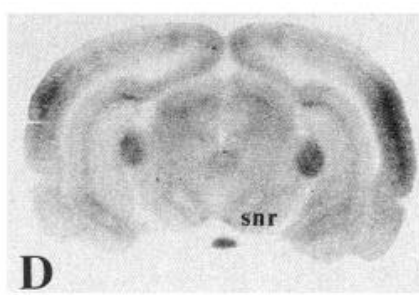

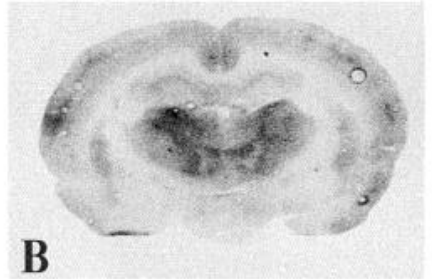

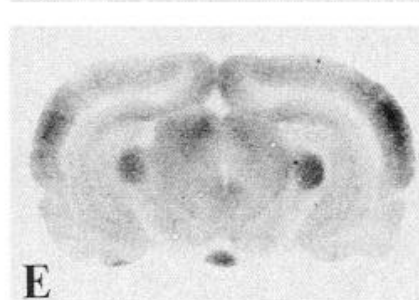

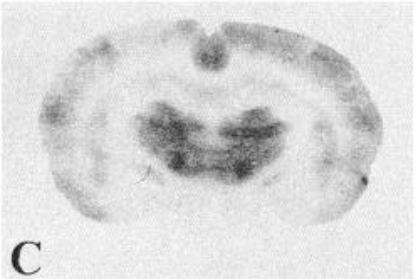

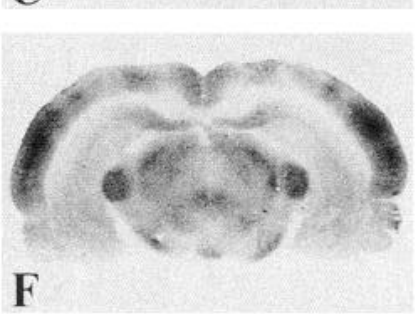

Figure 2. Challenge with $30 \mathrm{mg} / \mathrm{kg}$ cocaine following chronic treatment increases $\mathrm{rCMR}_{\text {elc }}$ in extrapyramidal efferent regions. Autoradiographs in $A-C$ illustrate the entopeduncular nucleus $(e p)$ and the adjacent lateral hypothalamus $(l h)$ at $2.3 \mathrm{~mm}$ posterior to bregma (Paxinos and Watson, 1986 ), while $D-F$ show the substantia nigra pars reticulata $(s n r)$ at $5.6 \mathrm{~mm}$ posterior to bregma (Paxinos and Watson, 1986). Compare the autoradiographs produced following saline treatment $(A$ and $D$ ) to those produced after chronic daily $10 \mathrm{mg} / \mathrm{kg}$ cocaine treatment following either $10 \mathrm{mg} / \mathrm{kg}(B$ and $E)$ or $30 \mathrm{mg} / \mathrm{kg}(C$ and $F)$ administered on the final treatment day. $\mathrm{rCMR}_{\mathrm{glc}}$ in the entopeduncular nucleus and substantia nigra pars reticulata increase significantly after challenge with $30 \mathrm{mg} / \mathrm{kg}$ cocaine. 
Table 1. Kegional cerebral metabolic rate $(\mu \mathrm{mol} / 100 \mathrm{gm} / \mathrm{min}$ glucose $\pm \mathrm{SEM})$ following 14 daily injections of saline vehicle or $10 \mathrm{mg} / \mathrm{kg}$ cocaine on the final $1,3,7,10$ or 14 treatment days

\begin{tabular}{|c|c|c|c|c|c|c|}
\hline Brain region & $\begin{array}{l}14 \text { days } \\
\text { saline } \\
(n-5)\end{array}$ & $\begin{array}{l}\text { Acute } \\
\text { cocaine } \\
(n-5)\end{array}$ & $\begin{array}{l}3 \text { days } \\
\text { cocaine } \\
(n-6)\end{array}$ & $\begin{array}{l}7 \text { days } \\
\text { cocaine } \\
(n-5)\end{array}$ & $\begin{array}{l}10 \text { days } \\
\text { cocaine } \\
(n-4)\end{array}$ & $\begin{array}{l}14 \text { days } \\
\text { cocaine } \\
(n-3)\end{array}$ \\
\hline \multicolumn{7}{|l|}{ Mesolimbic structures } \\
\hline Medial prefrontal cortex & $101.2 \pm 3.0$ & $99.2 \pm 7.4$ & $94.2 \pm 7.4$ & $108.2 \pm 4.1$ & $82.7 \perp 11.9$ & $80.7 \perp 12.0$ \\
\hline Nucleus accumbens, rostral pole & $92.8 \pm 4.3$ & $81.7 \pm 2.9$ & $84.7 \pm 5.9$ & $95.0 \pm 6.4$ & $78.7 \pm 10.6$ & $71.3 \pm 13.7$ \\
\hline Nucleus accumbens, core (rostral) & $82.8 \pm 3.4$ & $90.6 \pm 3.6$ & $83.2 \pm 6.0$ & $84.2 \pm 4.4$ & $62.0 \pm 7.4^{*} \dagger$ & $68.7 \pm 9.5^{* \dagger}$ \\
\hline Nucleus accumbens, core (caudal) & $78.6 \pm 3.1$ & $90.6 \pm 7.0$ & $81.7 \pm 4.9$ & $87.6 \pm 6.5$ & $65.5 \pm 9.2$ & $62.7 \pm 14.5$ \\
\hline Nucleus accumbens, shell (rostral) & $76.8 \pm 3.9$ & $86.8 \pm 2.1$ & $79.8 \pm 5.9$ & $80.2 \pm 3.8$ & $60.0 \pm 6.7^{* \dagger}$ & $65.3 \pm 10.1^{*} \dagger$ \\
\hline Nucleus accumbens, shell (caudal) & $69.4 \pm 1.9$ & $73.0 \pm 4.6$ & $69.2 \pm 5.6$ & $71.4 \pm 5.2$ & $57.2 \pm 6.5^{*} \dagger$ & $56.3 \pm 9.3$ \\
\hline Olfactory tubercle (rostral) & $100.4 \pm 3.8$ & $96.3 \pm 4.8$ & $98.8 \pm 7.2$ & $109.6 \pm 4.0$ & $82.7 \pm 13.1$ & $89.0 \pm 14.0$ \\
\hline Olfactory tubercle (mid) & $87.4 \pm 5.6$ & $94.2 \pm 2.5$ & $89.3 \pm 7.0$ & $94.6 \pm 4.4$ & $65.7 \pm 9.7^{*} \dagger$ & $77.0 \pm 7.0 \dagger$ \\
\hline Olfactory tubercle (caudal) & $81.0 \pm 3.4$ & $92.0 \pm 8.6$ & $89.7 \pm 5.3$ & $90.0 \pm 7.1$ & $65.5 \pm 8.5$ & $71.3 \pm 5.9$ \\
\hline Islands of Calleja (rostral) & $97.4 \pm 4.3$ & $97.7 \pm 5.0$ & $100.6 \pm 9.2$ & $107.2 \pm 4.5$ & $79.7 \pm 13.3$ & $87.3 \pm 10.4$ \\
\hline Islands of Calleja (caudal) & $80.6 \pm 4.8$ & $89.5 \pm 1.8$ & $86.0 \pm 8.1$ & $88.2 \pm 4.9$ & $64.7 \pm 11.0$ & $75.7 \pm 5.5$ \\
\hline Ventral tegmental area & $76.6 \pm 2.9$ & $73.0 \pm 3.9$ & $78.0 \pm 5.3$ & $78.6 \pm 5.3$ & $60.7 \pm 7.1$ & $70.0 \pm 6.5$ \\
\hline \multicolumn{7}{|l|}{ Extrapyramidal structures } \\
\hline Caudatoputamen, dorlat. (rostral) & $115.6 \pm 4.6$ & $116.0 \pm 4.9$ & $114.5 \pm 8.5$ & $120.2 \pm 5.9$ & $94.7 \pm 11.1$ & $80.5 \pm 9.7$ \\
\hline $\mathrm{CP}$, dorsolateral (mid) & $113.4 \pm 3.1$ & $119.4 \pm 7.6$ & $115.2 \pm 8.8$ & $125.0 \pm 8.6$ & $98.2 \pm 13.4$ & $80.5 \pm 9.6$ \\
\hline $\mathrm{CP}$, dorsolateral (caudal) & $104.8 \pm 2.4$ & $108.2 \pm 13.5$ & $109.2 \pm 8.7$ & $110.0 \pm 7.3$ & $96.5 \pm 10.7$ & $68.0 \pm 9.4$ \\
\hline $\mathrm{CP}$, ventromedial (rostral) & $110.6 \pm 5.5$ & $111.0 \pm 5.0$ & $109.3 \pm 7.9$ & $113.6 \pm 7.1$ & $88.7 \pm 10.8$ & $71.5 \pm 10.1^{*}+$ \\
\hline $\mathrm{CP}$, ventromedial (mid) & $108.4 \pm 3.2$ & $114.8 \pm 8.4$ & $106.0 \pm 10.3$ & $117.2 \pm 7.8$ & $90.2 \pm 13.1$ & $71.0 \pm 12.2$ \\
\hline$C P$, ventromedial (caudal) & $91.2 \pm 4.3$ & $90.2 \pm 10.2$ & $92.5 \pm 7.1$ & $92.5 \pm 6.7$ & $62.5 \pm 9.5$ & $47.0 \pm 14.0$ \\
\hline Globus pallidus & $69.0 \pm 4.0$ & $70.0 \pm 3.6$ & $74.3 \pm 6.5$ & $76.0 \pm 9.1$ & $62.5 \pm 9.5$ & $47.0 \pm 14.0$ \\
\hline Entopeduncular nucleus & $70.8 \pm 2.7$ & $82.0 \pm 5.5$ & $74.7 \pm 6.4$ & $81.8 \pm 6.9$ & $62.2 \pm 6.3$ & $70.0 \pm 11.2$ \\
\hline Subthalamus & $96.4 \pm 2.6$ & $93.4 \pm 8.9$ & $103.2 \pm 10.3$ & $101.2 \pm 7.2$ & $88.2 \pm 10.7$ & $74.0 \pm 10.5$ \\
\hline Substantia nigra pars compacta & $64.4 \pm 4.1$ & $70.2 \pm 4.6$ & $72.0 \pm 5.7$ & $72.2 \pm 3.6$ & $59.5 \pm 6.3$ & $63.7 \pm 11.1$ \\
\hline Substantia nigra pars reticulata & $78.0 \pm 2.7$ & $76.4 \pm 4.5$ & $80.2 \pm 5.9$ & $80.2 \pm 4.0$ & $63.2 \pm 6.0$ & $67.0 \pm 15.5$ \\
\hline \multicolumn{7}{|l|}{ Diencephalic structures } \\
\hline Medial habenula & $84.2 \pm 2.4$ & $78.8 \pm 3.6$ & $81.0 \pm 7.5$ & $79.8 \pm 5.9$ & $66.0 \pm 9.3$ & $67.3 \pm 9.6$ \\
\hline Lateral habenula (medial) & $96.8 \pm 3.3$ & $86.2 \pm 4.6$ & $89.3 \pm 7.4$ & $87.6 \pm 5.7$ & $76.2 \pm 9.9$ & $75.3 \pm 11.8$ \\
\hline Lateral habenula (lateral) & $107.6 \pm 3.0$ & $97.0 \pm 6.9$ & $99.8 \pm 7.8$ & $98.8 \pm 6.8$ & $84.7 \pm 10.7$ & $76.0 \pm 11.5$ \\
\hline Ventral thalamus (VPM/VPL) & $96.6 \pm 6.7$ & $97.2 \pm 5.7$ & $103.2 \pm 8.8$ & $108.6 \pm 8.1$ & $86.3 \pm 14.8$ & $89.7 \pm 15.1$ \\
\hline Laterodorsal thalamus & $107.0 \pm 3.9$ & $114.6 \pm 8.8$ & $115.5 \pm 10.4$ & $125.2 \pm 7.5$ & $90.5 \pm 14.4$ & $91.7 \pm 12.4$ \\
\hline Dorsomedial thalamus & $115.2 \pm 4.2$ & $128.2 \pm 7.0$ & $130.5 \pm 11.0$ & $132.0 \pm 8.7$ & $109.5 \pm 15.2$ & $96.0 \pm 16.6$ \\
\hline Ventral pallidum (whole) & $65.8 \pm 3.4$ & $69.2 \pm 7.9$ & $64.0 \pm 4.0$ & $71.2 \pm 6.8$ & $58.2 \pm 7.2$ & $55.0 \pm 11.9$ \\
\hline Substantia innominata & $64.0 \pm 3.8$ & $63.2 \pm 4.8$ & $62.5 \pm 5.0$ & $66.2 \pm 8.2$ & $54.0 \pm 8.2$ & $47.5 \pm 2.5$ \\
\hline Medial preoptic area & $58.2 \pm 3.5$ & $57.0 \pm 5.7$ & $54.7 \pm 5.0$ & $53.4 \pm 4.8$ & $45.2 \pm 6.4$ & $46.0 \pm 5.0$ \\
\hline Lateral preoptic area & $71.8 \pm 3.5$ & $75.2 \pm 5.8$ & $70.8 \pm 5.6$ & $77.2 \pm 8.1$ & $57.2 \pm 7.5$ & $53.0 \pm 9.3$ \\
\hline Lateral hypothalamus (rostral) & $73.0 \pm 0.5$ & $76.2 \pm 4.7$ & $69.7 \pm 4.8$ & $76.0 \pm 6.4$ & $57.5 \pm 6.2$ & $66.3 \pm 5.2$ \\
\hline Lateral hypothalamus (caudal) & $70.0 \pm 2.0$ & $69.8 \pm 5.0$ & $69.8 \pm 6.6$ & $72.4 \pm 5.2$ & $59.2 \pm 8.0$ & $50.0 \pm 12.8$ \\
\hline Arcuate nucleus & $50.5 \pm 4.9$ & $50.4 \pm 3.9$ & $46.5 \pm 4.8$ & $54.5 \pm 6.3$ & $37.7 \pm 3.8$ & $50.7 \pm 2.7$ \\
\hline \multicolumn{7}{|l|}{ Limbic regions } \\
\hline Medial septal nculeus & $89.2 \pm 1.8$ & $77.0 \pm 11.6$ & $86.2 \pm 6.0$ & $81.7 \pm 6.6$ & $67.7 \pm 6.6$ & $57.7 \pm 12.4$ \\
\hline Lateral septal nucleus & $53.6 \pm 2.4$ & $56.7 \pm 8.6$ & $55.2 \pm 4.6$ & $57.6 \pm 3.8$ & $49.5 \pm 6.6$ & $48.7 \pm 7.0$ \\
\hline Basolateral amygdala & $89.0 \pm 2.7$ & $97.2 \pm 5.6$ & $90.8 \pm 7.9$ & $92.6+6.7$ & $71.0+8.4 \dagger$ & $58.0+9.9 * \dagger$ \\
\hline Central amygdala & $60.0 \pm 2.3$ & $65.2 \pm 3.8$ & $58.5 \pm 4.8$ & $61.8 \pm 6.3$ & $48.2 \pm 5.4$ & $52.5 \pm 8.5$ \\
\hline Piriform cortex (rostral) & $119.0 \pm 5.4$ & $113.0 \pm 5.1$ & $123.0 \pm 10.1$ & $130.6 \pm 9.5$ & $101.2 \pm 15.3$ & $99.0 \pm 11.3$ \\
\hline Piriform cortex (mid) & $117.2 \pm 8.0$ & $120.8 \pm 5.7$ & $116.3 \pm 7.3$ & $123.4 \pm 8.5$ & $85.5 \pm 12.1^{*} \dagger$ & $92.6 \pm 10.0^{*} \dagger$ \\
\hline Piriform cortex (caudal) & $108.2 \pm 4.4$ & $120.6 \pm 6.1$ & $115.2 \pm 6.5$ & $127.8 \pm 10.0$ & $87.0 \pm 13.3$ & $88.3 \pm 17.7$ \\
\hline Cingulate cortex (rostral) & $103.2 \pm 4.8$ & $100.8 \pm 4.6$ & $99.2 \pm 6.7$ & $107.2 \pm 6.1$ & $83.5 \pm 11.4$ & $66.0 \pm 12.4^{*} \dagger$ \\
\hline Cingulate cortex (caudal) & $100.2 \pm 3.5$ & $100.2 \pm 7.1$ & $97.3 \pm 7.3$ & $105.8 \pm 7.5$ & $82.5 \pm 13.0$ & $77.0 \pm 13.1$ \\
\hline Entorhinal cortex (superficial) & $81.8 \pm 6.7$ & $77.5 \pm 4.8$ & $88.2 \pm 6.5$ & $76.0 \pm 5.7$ & $62.7 \pm 9.3$ & $78.0 \pm 4.5$ \\
\hline Entorhinal cortex (deep) & $87.0 \pm 9.1$ & $87.2 \pm 5.7$ & $92.8 \pm 7.9$ & $74.2 \pm 3.6$ & $67.7 \pm 8.7$ & $78.0 \pm 6.7$ \\
\hline \multicolumn{7}{|l|}{ Cortical regions } \\
\hline Agranular motor cortex & $95.8 \pm 3.4$ & $104.0 \pm 8.4$ & $102.5 \pm 6.4$ & $106.2 \pm 6.3$ & $78.7 \pm 10.1$ & $72.0 \pm 9.4$ \\
\hline Somatosensory cortex & $97.4 \pm 3.5$ & $99.8 \pm 7.7$ & $100.0 \pm 7.2$ & $105.2 \pm 7.9$ & $79.2 \pm 10.8$ & $71.0 \pm 8.0$ \\
\hline Visual cortex & $103.4 \pm 5.1$ & $101.4 \pm 7.4$ & $103.5 \pm 8.1$ & $105.4 \pm 4.8$ & $90.7 \pm 11.0$ & $78.5 \pm 13.5$ \\
\hline Auditory cortex & $138.8 \pm 10.4$ & $136.8 \pm 7.5$ & $134.5+11.4$ & $138.2 \pm 11.6$ & $110.7 \pm 14.1$ & $114.0 \pm 7.1$ \\
\hline
\end{tabular}


Table 1. Continued.

\begin{tabular}{|c|c|c|c|c|c|c|}
\hline Brain region & $\begin{array}{l}14 \text { days } \\
\text { saline } \\
(n=5)\end{array}$ & $\begin{array}{l}\text { Acute } \\
\text { cocaine } \\
(n=5)\end{array}$ & $\begin{array}{l}3 \text { days } \\
\text { cocaine } \\
(n=6)\end{array}$ & $\begin{array}{l}7 \text { days } \\
\text { cocaine } \\
(n=5)\end{array}$ & $\begin{array}{l}10 \text { days } \\
\text { cocaine } \\
(n=4)\end{array}$ & $\begin{array}{l}14 \text { days } \\
\text { cocaine } \\
(n=3)\end{array}$ \\
\hline \multicolumn{7}{|l|}{ Brainstem and other regions } \\
\hline Periaqueductal gray matter & $75.6 \pm 4.3$ & $80.4 \pm 4.6$ & $78.2 \pm 5.8$ & $82.2 \pm 6.2$ & $66.7 \pm 8.7$ & $67.0 \pm 13.1$ \\
\hline Superior colliculus & $84.4 \pm 5.9$ & $86.2 \pm 4.1$ & $82.0 \pm 7.4$ & $93.6 \pm 7.8$ & $65.2 \pm 10.3$ & $66.0 \pm 8.9$ \\
\hline Dorsal raphe nuclcus & $90.4 \pm 10.9$ & $90.7 \pm 11.2$ & $92.2 \pm 3.5$ & $99.0 \pm 7.1$ & $77.0 \pm 10.3$ & $85.0 \pm 3.0$ \\
\hline Inferior colliculus & $152.2 \pm 9.2$ & $133.8 \pm 11.6$ & $123.7 \pm 5.5$ & $127.2 \pm 8.7$ & $102.7 \pm 16.5$ & $127.0 \pm 8.0$ \\
\hline Cerebellar cortex & $62.8 \pm 2.8$ & $66.2 \pm 4.2$ & $77.3 \pm 6.3$ & $68.0 \pm 3.3$ & $52.7 \pm 6.8$ & $53.7 \pm 9.5$ \\
\hline Locus ceruleus & $114.8 \pm 13.2$ & $105.2 \pm 9.4$ & $117.4 \pm 5.7$ & $100.2 \pm 7.4$ & $82.7 \pm 11.5$ & $83.3 \pm 16.2$ \\
\hline Superior olive & $115.8 \pm 8.3$ & $117.6 \pm 11.3$ & $108.0 \pm 7.6$ & $123.0 \pm 13.9$ & $100.2 \pm 13.5$ & $93.0 \pm 14.5$ \\
\hline Corpus callosum & $39.0 \pm 4.3$ & $40.2 \pm 3.2$ & $37.8 \pm 6.4$ & $38.2 \pm 3.6$ & $36.0 \pm 5.2$ & $46.7 \pm 6.3$ \\
\hline
\end{tabular}

* Significantly $(p \leq 0.05)$ different compared to saline treatment by one-way ANOVA followed by Newman-Keuls post hoc test.

$\dagger$ Significantly $(p \leq 0.05)$ different compared to acute $10 \mathrm{mg} / \mathrm{kg}$ cocaine treatment by ANOVA folowed by Newman-Keuls test.

inhibitory effect of cocaine on dopamine uptake in the NAC (Izenwasser and Cox, 1992), while repeated treatment at high levels reduced ${ }^{3} \mathrm{H}$-mazindol binding to dopamine transporters in the NAc during subsequent cocaine withdrawal (Sharpe et al., 1991). Furthermore, studies of immediate-early gene expression reveal that the levels of NAc c-fos and c-jun mRNA do not change in response to cocaine challenge following chronic treatment (Hope et al., 1992), and zif268 mRNA basal levels are suppressed in response to cocaine challenge after chronic treatment (Bhat et al., 1992). In contrast, acute cocaine treatment induced expression of each of these immediate-early genes (Hope et al., 1992; Moratalla et al., 1992, 1993). Thus, the induction of immediate-early gene expression reflecting cellular activity also becomes tolerant in response to chronic cocaine treatment.

The present data show that regional brain metabolic activity exhibits a time-dependent pattern in response to repeated daily treatment with $10 \mathrm{mg} / \mathrm{kg}$ cocaine (Table 1). Whereas acute treatment slightly increased $\mathrm{rCMR}_{\mathrm{glc}}$, subsequent treatment had no effect until more than $7 \mathrm{~d}$ of treatment, whereupon the same cocaine dose reduced rCMR $_{\text {glc }}$ in mesolimbic forebrain compared to saline treatment. This gradual shift from initial activation to eventual reduction of $\mathrm{rCMR}_{\mathrm{glc}}$ after chronic cocaine treatment could represent tolerance of metabolic response to cocaine in selective brain regions. However, the exact time course and magnitude of these effects could be dependent upon the specific dose and paradigm of drug administration.

rCMR $_{\text {glc }}$ in the NAc shell, wherein dopamine turnover is increased by stress (Deutch and Cameron, 1992), was reduced by chronic $10 \mathrm{mg} / \mathrm{kg}$ cocaine treatment in both experiments (Tables $1,2)$. The NAc shell receives afferent projections from the $\mathrm{MPf}_{\mathrm{il}}$ (Berendse et al., 1992), which also exhibited reduced $\mathrm{rCMR}_{\mathrm{glc}}$ upon selective regional analysis (Table 2). Glucose utilization is known to be relatively greater in synaptic terminals than in somata due to their increased surface-to-volume ratio (Schwartz et al., 1979; Kadekaro et al., 1987). Therefore, a specific mesocorticolimbic forebrain circuit extending from the $\mathrm{MPF}_{\mathrm{i}}$ to the NAc shell might be preferentially affected, exhibiting reduced activity following chronic cocaine treatment. In fact, projections from the NAc shell to the sublenticular extended amygdala (Heimer et al., 1991) have been implicated in the modulation of cocaine reinforcement (Robledo and Koob, 1993). The olfactory tubercle, in which $\mathrm{rCMR}_{\mathrm{glc}}$ may be related to cocaine reinforcement or expression of stereotypic behavior (Kornetsky et al., 1991), consistently exhibited reduced rCMR $_{\text {glc }}$ after chronic cocaine treatment. The ABL, which together with the NAc may be necessary for expression of locomotor sensitization (Post et al., 1987), and the PCalso exhibit consistent metabolic deficits compared to chronic saline treatment. The NAc core, the rostral portion of which was affected by chronic cocaine treatment ( Table 1), innervates the SNc (Zahm and Brog, 1992), wherein $\mathrm{rCMR}_{\mathrm{glc}}$ was reduced by chronic cocaine treatment. Few other extrapyramidal regions showed any reduction of $\mathrm{rCMR}_{\mathrm{glc}}$ following chronic cocaine treatment. Interestingly, $\mathrm{rCMR}_{\mathrm{glc}}$ in the LC, a source of noradrenergic innervation, was also reduced after chronic cocaine treatment in experiment 2 , revealing a potential effect on synaptic activity in this brain region. These results suggest that the cocaine-induced reduction of metabolic activity, which was largely limited to mesocorticolimbic brain regions, might be related to stereotypy and drug reinforcement regulated by these circuits rather than to motor output, unless such locomotor activity is also affected by mesolimbic projections. Such regional reduction could function to decrease the expression of these behaviors. Alternatively, the same level of behavioral expression might require less synaptic stimulus following chronic drug treatment. Further studies that correlate bchavioral and metabolic response are required to elucidate this question.

It should be noted that these experiments examined $\mathrm{rCMR}_{\mathrm{glc}}$ response to cocaine treatment, rather than basal metabolic activity present prior to terminal drug treatment. Therefore, we cannot determine whether the final dose of $10 \mathrm{mg} / \mathrm{kg}$ cocaine actually increased or decreased $\mathrm{rCMR}_{\mathrm{glc}}$ compared to basal values. The resulting $\mathrm{rCMR}_{\mathrm{glc}}$ values were significantly reduced compared to regional values from saline-treated animals, which could occur either by a reduction of basal $\mathrm{rCMR}_{\mathrm{glc}}$, a reduction of $\mathrm{rCMR}_{\mathrm{glc}}$ in response to cocaine treatment, or both. Previous studies have shown that $\mathrm{rCMR}_{\mathrm{glc}}$ is reduced in mesolimbic forebrain at various times during withdrawal following chronic contingent (Hammer et al., 1993) and noncontingent (Clow and Hammer, 1991) cocaine treatment. Since the basal metabolic state prior to terminal cocaine treatment resembles that of 24 
Table 2. Regional cereloral metalolic rate $(\mu \mathrm{mol} / 100 \mathrm{gm} / \mathrm{min}$ glucose \pm SEM) following repeated daily saline or $10 \mathrm{mg} / \mathrm{kg}$ cocaine injection with or without $30 \mathrm{mg} / \mathrm{kg}$ cocaine treatment on the final day

\begin{tabular}{|c|c|c|c|}
\hline Brain region & $\begin{array}{l}\text { Saline } \\
(n=5)\end{array}$ & $\begin{array}{l}\text { Chronic cocaine } \\
\text { (daily } 10 \mathrm{mg} / \mathrm{kg} \\
\text { treatment) } \\
(n=6)\end{array}$ & $\begin{array}{l}\text { Challenge with } \\
30 \mathrm{mg} / \mathrm{kg} \text { after } \\
\text { chronic } \\
\text { treatment } \\
(n=7)\end{array}$ \\
\hline \multicolumn{4}{|l|}{ Mesolimbic structures } \\
\hline Medial prefrontal cx., prelimbic & $113.8 \pm 5.0$ & $102.7 \pm 5.0$ & $101.7 \pm 3.1$ \\
\hline Medial prefrontal cx., infralimbic & $98.2 \pm 4.9$ & $85.2 \pm 3.5^{*}$ & $87.3 \pm 2.0^{*}$ \\
\hline Nucleus accumbens, rostral pole & $100.4 \pm 5.1$ & $93.2 \pm 5.1$ & $101.4 \pm 2.6$ \\
\hline Nucleus accumbens, core (rostral) & $97.4 \pm 5.0$ & $84.0 \pm 4.1$ & $87.6 \pm 3.8$ \\
\hline Nucleus accumbens, core (caudal) & $89.0 \pm 5.1$ & $78.0 \pm 4.2$ & $83.4 \pm 4.1$ \\
\hline Nucleus accumbens, shell (rostral) & $98.2 \pm 5.2$ & $82.7 \pm 4.6^{*}$ & $81.4 \pm 4.9$ \\
\hline Nucleus accumbens, shell (caudal) & $80.2 \pm 3.9$ & $67.8 \pm 1.7^{*}$ & $71.6 \pm 2.8$ \\
\hline Olfactory tubercle (rostral) & $123.8 \pm 6.1$ & $110.5 \pm 5.4$ & $112.6 \pm 4.2$ \\
\hline Olfactory tubercle (mid) & $109.4 \pm 4.0$ & $94.5 \pm 4.9^{*}$ & $101.6 \pm 5.0$ \\
\hline Olfactory tubercle (caudal) & $99.2 \pm 4.5$ & $89.7 \pm 3.4$ & $95.3 \pm 4.1$ \\
\hline Islands of Calleja (rostral) & $121.8 \pm 4.7$ & $108.7 \pm 4.0$ & $114.9 \pm 4.9$ \\
\hline Islands of Calleja (caudal) & $104.2 \pm 4.6$ & $90.3 \pm 4.3$ & $97.4 \pm 5.2$ \\
\hline Ventral tegmental area & $86.8 \pm 3.6$ & $83.5 \pm 5.7$ & $98.6 \pm 3.1$ \\
\hline \multicolumn{4}{|l|}{ Extrapyramidal structures } \\
\hline Caudatoputamen (CP) dorsolateral (rostral) & $117.6 \pm 4.3$ & $101.5 \pm 5.3^{*}$ & $114.1 \pm 3.5$ \\
\hline $\mathrm{CP}$, dorsolateral (mid) & $116.4 \pm 4.4$ & $103.0 \pm 4.3^{*}$ & $118.6 \pm 3.4 \dagger$ \\
\hline $\mathrm{CP}$, dorsolateral (caudal) & $109.8 \pm 3.7$ & $97.7 \pm 5.1$ & $117.7 \pm 4.3 \dagger$ \\
\hline $\mathrm{CP}$, ventromedial (rostral) & $114.2 \pm 4.2$ & $99.7 \pm 5.7$ & $109.1 \pm 3.3$ \\
\hline $\mathrm{CP}$, ventromedial (mid) & $111.6 \pm 4.3$ & $100.3 \pm 3.3$ & $111.6 \pm 2.0 \dagger$ \\
\hline $\mathrm{CP}$, ventromedial (caudal) & $98.8 \pm 2.9$ & $90.7 \pm 3.7$ & $102.0 \pm 2.8$ \\
\hline Globus pallidus & $75.0 \pm 4.0$ & $69.0 \pm 4.6$ & $86.9 \pm 2.5^{* \dagger}$ \\
\hline Entopeduncular nucleus & $80.2 \pm 3.8$ & $69.3 \pm 5.8$ & $91.6 \pm 3.4 \dagger$ \\
\hline Subthalamus (rostral) & $96.0 \pm 4.7$ & $88.8 \pm 6.4$ & $98.6 \pm 4.2$ \\
\hline Subthalamus (caudal) & $97.6 \pm 4.5$ & $92.2 \pm 6.6$ & $114.3 \pm 4.5^{* \dagger}$ \\
\hline Substantia nigra pars compacta & $86.8 \pm 4.0$ & $75.5 \pm 3.3^{*}$ & $88.6 \pm 3.4 \dagger$ \\
\hline Substantia nigra pars reticulata & $76.2 \pm 4.7$ & $69.5 \pm 4.6$ & $98.9 \pm 4.7^{*} \dagger$ \\
\hline \multicolumn{4}{|l|}{ Dicncephalic structures } \\
\hline Medial habenula & $97.4 \pm 4.7$ & $76.3 \pm 5.8^{*}$ & $86.3 \pm 4.1$ \\
\hline Lateral habenula (medial) & $109.0 \pm 5.3$ & $84.2 \pm 6.2^{*}$ & $86.0 \pm 4.4^{*}$ \\
\hline Lateral habenula (lateral) & $118.4 \pm 4.8$ & $88.3 \pm 6.8^{*}$ & $90.4 \pm 4.7^{*}$ \\
\hline Ventral thalamus (VPM/VPL) & $109.6 \pm 6.2$ & $92.5 \pm 6.2$ & $107.4 \pm 4.6$ \\
\hline Laterodorsal thalamus & $117.4 \pm 5.4$ & $102.0 \pm 8.4$ & $122.0 \pm 5.7$ \\
\hline Dorsomedial thalamus & $115.6 \pm 4.6$ & $105.5 \pm 7.7$ & $121.6 \pm 5.5$ \\
\hline Ventral pallidum, dorsolateral & $75.8 \pm 4.3$ & $65.8 \pm 2.8$ & $76.7 \pm 3.0$ \\
\hline Ventral pallidum, ventromedial & $82.4 \pm 5.7$ & $70.3 \pm 4.0$ & $79.0 \pm 2.9$ \\
\hline Substantia innominata & $67.0 \pm 3.6$ & $59.3 \pm 3.9$ & $63.7+2.0$ \\
\hline Diagonal band, horizontal limb & $87.6 \pm 4.4$ & $82.8 \pm 4.6$ & $82.1 \pm 2.8$ \\
\hline Bed nucleus of the stria terminalis & $63.2 \pm 3.3$ & $55.2 \pm 3.2$ & $58.9 \pm 2.2$ \\
\hline Medial preoptic area & $66.6 \pm 3.5$ & $56.5 \pm 2.8$ & $60.9 \pm 1.9$ \\
\hline Lateral preoptic area & $86.0 \pm 7.5$ & $71.0 \pm 4.7$ & $77.9 \pm 3.2$ \\
\hline Lateral hypothalamus (rostral) & $82.2 \pm 2.9$ & $69.8 \pm 4.9$ & $76.4 \pm 3.8$ \\
\hline Lateral hypothalamus (caudal) & $78.2 \pm 3.6$ & $71.8 \pm 5.1$ & $76.0 \pm 2.8$ \\
\hline$\Lambda$ rcuate nucleus & $63.0 \pm 3.2$ & $56.8 \pm 4.4$ & $63.3 \pm 2.1$ \\
\hline
\end{tabular}

hr cocaine abstinence, it is likely that basal $\mathrm{rCMR}_{\mathrm{glc}}$ was reduced following chronic cocaine treatment.

\section{Effect of subsequent cocaine challenge}

We observed that $\mathrm{rCMR}_{\mathrm{glc}}$ increased following $30 \mathrm{mg} / \mathrm{kg}$ cocaine challenge in CP, GP, EP, STh, and SNr, as well as in sensorimotor cortex, PAG, and LC compared to chronic treatment with $10 \mathrm{mg} / \mathrm{kg}$ cocaine alone. Moreover, $\mathrm{rCMR}_{\mathrm{glc}}$ was significantly greater after $30 \mathrm{mg} / \mathrm{kg}$ cocaine challenge than after chronic saline treatment in GP, STh, and SNr, as has been observed in these regions following acute cocaine treatment with the same cocaine dose (London et al., 1986). Therefore, striatal efferent neurons might be activated in response to cocaine challenge, since these neurons innervate the affected extrapyramidal regions. 


\begin{tabular}{|c|c|c|c|}
\hline Brain region & $\begin{array}{l}\text { Saline } \\
(n=5)\end{array}$ & $\begin{array}{l}\text { Chronic cocaine } \\
\text { (daily } 10 \mathrm{mg} / \mathrm{kg} \\
\text { treatment) } \\
(n=6)\end{array}$ & $\begin{array}{l}\text { Challenge with } \\
30 \mathrm{mg} / \mathrm{kg} \text { after } \\
\text { chronic } \\
\text { treatment } \\
(n=7)\end{array}$ \\
\hline \multicolumn{4}{|l|}{ Limbic regions } \\
\hline Medial septal nucleus & $82.2 \pm 5.0$ & $77.5 \pm 4.3$ & $82.0 \pm 2.5$ \\
\hline Lateral septal nculeus & $71.4 \pm 2.0$ & $67.2 \pm 4.7$ & $81.6 \pm 4.2$ \\
\hline Basolateral amygdala & $103.8 \pm 3.9$ & $84.5 \pm 5.6^{*}$ & $92.7 \pm 3.8$ \\
\hline Central amygdala & $69.4 \pm 3.6$ & $56.3 \pm 3.6^{*}$ & $63.4 \pm 2.4$ \\
\hline Piriform cortex (rostral) & $135.0 \pm 5.1$ & $122.0 \pm 6.9$ & $125.6 \pm 3.4$ \\
\hline Piriform cortex (mid) & $128.6 \pm 4.1$ & $112.3 \pm 5.4^{*}$ & $117.6 \pm 3.6$ \\
\hline Piriform cortex (caudal) & $125.6 \pm 4.7$ & $105.8 \pm 6.1^{*}$ & $118.3 \pm 3.4$ \\
\hline Cingulate cortex (rostral) & $114.6 \pm 5.2$ & $100.7 \pm 5.7$ & $103.9 \pm 2.4$ \\
\hline Cingulate cortex (caudal) & $108.8 \pm 5.2$ & $95.0 \pm 3.9$ & $101.7 \pm 1.7$ \\
\hline Entorhinal cortex (superficial) & $86.8 \pm 4.5$ & $82.2 \pm 4.0$ & $89.3 \pm 2.8$ \\
\hline Entorhinal cortex (deep) & $87.6 \pm 5.1$ & $79.3 \pm 4.1$ & $87.7 \pm 2.5$ \\
\hline \multicolumn{4}{|l|}{ Cortical regions } \\
\hline Agranular motor cortex & $102.8 \pm 3.6$ & $91.8 \pm 4.8$ & $109.4 \pm 2.9 \dagger$ \\
\hline Somatosensory cortex & $105.8 \pm 4.1$ & $89.0 \pm 3.2^{*}$ & $100.7 \pm 2.5 \dagger$ \\
\hline Visual cortex & $102.8 \pm 6.4$ & $92.0 \pm 5.8$ & $109.4 \pm 4.0$ \\
\hline Auditory cortex & $129.8 \pm 4.5$ & $122.0 \pm 5.8$ & $136.4 \pm 4.2$ \\
\hline \multicolumn{4}{|l|}{ Brainstem and other regions } \\
\hline Medial geniculate nucleus & $125.0 \pm 6.0$ & $112.3 \pm 3.3$ & $118.6 \pm 7.2$ \\
\hline Periaqueductal gray matter & $84.2 \pm 4.9$ & $77.0 \pm 2.9$ & $88.6 \pm 1.7 \dagger$ \\
\hline Superior colliculus & $94.4 \pm .2 .7$ & $90.7 \pm 4.1$ & $100.0 \pm 2.5$ \\
\hline Dorsal raphe nucleus & $90.6 \pm 6.2$ & $81.3 \pm 4.3$ & $89.4 \pm 3.4$ \\
\hline Median raphe nucleus & $99.4 \pm 9.6$ & $80.5 \pm 5.6$ & $86.7 \pm 6.4$ \\
\hline Inferior colliculus & $138.8 \pm 3.3$ & $119.2 \pm 4.6^{*}$ & $121.0 \pm 3.8^{*}$ \\
\hline Cerebellar cortex & $72.2 \pm 4.8$ & $68.3 \pm 7.8$ & $83.9 \pm 3.4$ \\
\hline Locus ceruleus & $123.0 \pm 5.6$ & $98.5 \pm 4.2^{*}$ & $117.3 \pm 6.8 \dagger$ \\
\hline Superior olive & $128.2 \pm 3.2$ & $103.0 \pm 5.8^{*}$ & $106.2 \pm 5.8^{*}$ \\
\hline Corpus callosum & $39.8 \pm 3.0$ & $37.5 \pm 3.6$ & $41.0 \pm 3.3$ \\
\hline
\end{tabular}

* Significantly $(p \leq 0.05)$ different compared to saline treatment by Duncan's multiple range test.

$\dagger$ Significantly $(p \leq 0.05)$ different compared to chronic $10 \mathrm{mg} / \mathrm{kg}$ cocaine treatment by Duncan's multiple range test.

It is known, for example, that c-fos expression is induced by acute cocaine in striatal neurons (Moratalla et al., 1993), and this effect is blocked by dopamine $D_{1}$ antagonist pretreatment (Young et al., 1991). Expression of c-fos is thought to be related to neuronal activity as illustrated by local 2DG uptake (Sharp et al., 1989), although additional factors might induce c-fos expression without affecting neuronal activity. Since the striatal neurons that project to GP, EP, and SNr (Kawaguchi et al., 1990) probably contain $D_{1}$ receptors (Gerfen et al., 1990), their activation by cocaine could increase $\mathrm{rCMR}_{\text {glc }}$ in regions containing their efferent terminals. Such cocaine-induced activation might also involve synergistic regulation of both $D_{1}$ and $D_{2}$ receptors, as treatment of intact rats with a selective dopamine $\mathrm{D}_{1}$ agonist increases $\mathrm{rCMR}_{\mathrm{glc}}$ only in $\mathrm{SNr}$ (Trugman and James, 1993).

Although challenge with $30 \mathrm{mg} / \mathrm{kg}$ cocaine increased $\mathrm{rCMR}_{\mathrm{glc}}$ in extrapyramidal regions, such treatment had little effect in mesolimbic structures. Instead, $\mathrm{rCMR}_{\mathrm{glc}}$ remained depressed in the NAc shell and was significantly reduced in $\mathrm{MPf}_{\mathrm{il}}$ after 30 $\mathrm{mg} / \mathrm{kg}$ cocaine treatment compared to saline treatment. The present study did not compare the regional metabolic response of cocaine challenge to that of acute administration; however, several studies using the acute paradigm have reported similar results to those observed herein. Acute intraperitoneal treatment with $30 \mathrm{mg} / \mathrm{kg}$ cocaine did not affect $\mathrm{rCMR}_{\text {glc }}$ in mesolimbic regions (London et al., 1986, 1990), although acute intravenous cocaine increased rCMR $\mathrm{glc}_{\mathrm{g}}$ in the MPf and NAc (Porrino et al., 1988). Hence, the route of administration might contribute to the magnitude and location of metabolic effects. The preferential activation of extrapyramidal efferent circuits by cocaine challenge following chronic treatment might be related to cocaineinduced locomotor activity (i.e., sensitization). This relationship is complex, however, since treatment with $30 \mathrm{mg} / \mathrm{kg}$ cocaine increased $\mathrm{rCMR}_{\mathrm{glc}}$ only in the $\mathrm{SNr}$ of naive, gallamine-paralyzed animals (London et al., 1990). In any case, this effect seems unrelated to the prior induction of metabolic tolerance in mesolimbic circuits.

Challenge with $30 \mathrm{mg} / \mathrm{kg}$ cocaine also produced metabolic activation in primary somatosensory and motor cortices and LC. Cortical activation could he a consequence of the increased motor activity (and sensory feedback) observed. It is possible that cocaine has direct effects on synaptic terminals in the LC, and this could be related to the effects of this drug on noradrenergic transporters. The reduction of $\mathrm{rCMR}_{\mathrm{glc}}$ in the $\mathrm{LH}$ fol- 
lowing $30 \mathrm{mg} / \mathrm{kg}$ cocaine challenge is consistent with the effect on this brain region of the same dose administered acutely (London et al., 1986). Repeated administration of $10 \mathrm{mg} / \mathrm{kg}$ cocaine reduced $\mathrm{rCMR}_{\mathrm{glc}}$ (Table 2 ), and subsequent challenge with 30 $\mathrm{mg} / \mathrm{kg}$ cocaine produced no further change. The $\mathrm{LH}$ receives extensive projections from the EP (Herkenham and Nauta, 1977; van der Kooy and Carter, 1981) as well as dopamine innervation from the medial VTA (Skagerberg et al., 1984). Thus, cocaineinduced activation of GABAergic terminals in EP could inhibit firing of neurons projecting to the habenula, or dopamine could have direct effects on synaptic activity, either of which could result in reduced $\mathrm{rCMR}_{\mathrm{glc}}$ in this region.

\section{Mechanisms of chronic cocaine effects}

Although the cause of these differential metabolic responses following chronic treatment is unclear, the induced reduction of $\mathrm{rCMR}_{\mathrm{glc}}$ apparently does not involve a reduction of regional drug availability. In fact, pharmacokinetic analyses show that regional brain cocaine concentration is elevated after chronic treatment (Pettit et al., 1990; Cass and Zahniser, 1993), perhaps due to increased absorption of the drug following intraperitoneal injection (Pan et al., 1991). Cocaine levels are higher in the NAc than in striatum during the first 20 min following a $10 \mathrm{mg} / \mathrm{kg}$ intraperitoneal injection (Javaid and Davis, 1993), which represents the period during which most uptake of 2DG and its intracellular trapping in the form of 2DG-6-phosphate occur (Sokoloff et al., 1977). Thus, the relatively greater effect of cocaine challenge on $\mathrm{rCMR}_{\mathrm{glc}}$ in extrapyramidal than mesolimbic regions is probably not due to higher striatal cocaine levels.

The effect of chronic cocaine on $\mathrm{rCMR}_{\mathrm{glc}}$ resembles the pattern of altered mesolimbic dopamine response to chronic cocaine. Repeated daily intraperitoneal administration of $10 \mathrm{mg} / \mathrm{kg}$ cocaine for $10 \mathrm{~d}$ has been shown to increase basal dopamine level in the NAc while attenuating the relative dopamine response to cocaine challenge (Weiss et al., 1992), which could further reduce NAc neuronal firing, thereby decreasing $\mathrm{rCMR}_{\mathrm{glc}}$ in regions containing the efferent terminals of NAc neurons, since iontophoretic application of either dopamine (Henry et al., 1989) or dopamine $\mathrm{D}_{1}$ agonists (Henry and White, 1991) reportedly enhances inhibition of NAc neuronal firing in anesthetized animals after repeated cocaine treatment. This may occur even in the absence of increased $D_{1}$ receptor number (Kleven et al., 1990; Peris et al., 1990; Mayfield et al., 1992) due to a postreceptor increase of adenylate cyclase and cAMP-dependent protein kinase activity in NAc (Terwilliger et al., 1991). However, the predominant inhibitory action of dopamine on NAc neurons in anesthetized animals might not occur in freely moving animals, which exhibit dopaminergic excitation in the NAc (West et al., 1992). Moreover, the effect of chronic cocaine on basal dopamine level is somewhat controversial, as $10 \mathrm{mg} / \mathrm{kg}$ administered twice daily reportedly increases basal dopamine level in NAc over the first $3 \mathrm{~d}$ before returning to baseline on days 4 and 5 , and significantly decreases NAc basal dopamine level thereafter (Imperato et al., 1992). Indeed, this putative time course of altered basal dopamine level is remarkably similar to that of $\mathrm{rCMR}_{\mathrm{glc}}$ which we observed in the same region after repcated treatment. It is possible that the resulting dopamine basal level affects the activity of cortical neurons that project to the NAc or of local circuit neurons located therein, thus altering $\mathrm{rCMR}_{\mathrm{glc}}$ in these sites.

Although few previous studies have examined $\mathrm{rCMR}_{\mathrm{glc}}$ response to chronic cocaine treatment in experimental animals, recent positron emission tomographic studies using ${ }^{18} \mathrm{~F}-2 \mathrm{DG}$ in humans show that $\mathrm{rCMR}_{\mathrm{gic}}$ decreases in the cerebral cortex, basal ganglia, and thalamus following cocaine administration in cocaine abusers (London et al., 1990). Cocaine administration also reduced regional cerebral blood flow, which is tightly coupled to $\mathrm{rCMR}_{\mathrm{glc}}$ even after cocaine administration (Sharkey et al., 1991), in frontal cortex and basal ganglia of cocaine abusers (Pearlson et al., 1993). These results are consistent with a differential effect of cocaine on $\mathrm{rCMR}_{\mathrm{glc}}$ in naive subjects versus those experienced with cocaine, as we have observed in animals. Thus, animal studies would appear to provide an appropriate model for human brain response to chronic cocaine administration. Furthermore, the results suggest that regional metabolic responses to cocaine administration in naive subjects might be quite different from those observed in cocaine (or polydrug) abusers. The resulting $\mathrm{rCMR}_{\mathrm{glc}}$ pattern suggests that chronic use can produce tolerance to the acute metabolic response. Moreover, the cellular, chemical, and molecular alterations that contribute to this regional metabolic response apparently last for days to weeks after cessation of cocaine self-administration, in both rats (Hammer et al., 1993) and humans (Volkow et al., 1992).

\section{References}

Berendse HW, Galis-de Graaf Y, Groenewegen HJ (1992) Topographical organization and relationship with ventral striatal compartments of prefrontal corticostriatal projections in the rat. J Comp Neurol 316 : 314-347.

Bhat RV, Cole $\Lambda$ J, Baraban JM (1992) Chronic cocaine treatment suppresses basal expression of zif268 in rat forebrain: in situ hybridization studies. J Pharmacol Exp Ther 263:343-349.

Cass WA, Zahniser NR (1993) Cocaine levels in striaturn and nucleus accumbens: augmentation following challenge injection in rats withdrawn from repeated cocaine administration. Neurosci Lett 152:177180.

Clow DW, Hammer RP (1991) Cocaine abstinence following chronic treatment alters cerebral metabolism in dopaminergic reward regions: bromocriptine enhances recovery. Neuropsychopharmacology 4:7175.

Crane AL, Porrino IJ (1989) Adaptation of the quantitative $2\left[{ }^{14} \mathrm{C}\right]$ deoxyglucose method for use in freely moving rats. Brain Res 499:87-92.

Deutch AY, Cameron DS (1992) Pharmacological characterization of dopamine systems in the nucleus accumbens core and shell. Neuroscience 46:49-56.

Emmett-Oglesby MW, Lane JD (1992) Tolerance to the reinforcing effects of cocaine. Behav Pharmacol 3:193-200.

Emmett-Oglesby MW, Peltier RL, Depoortere RY, Pickering CL, Hooper ML, Gong YH, Lane JD (1993) Tolerance to self-administration of cocaine in rats: time course and dose-response determination using a multi-dose method. Drug Alcohol Depend 32:247-256.

Gerfen CR, Engber TM, Mahan LC, Susel Z, Chase TN, Monsma FJ, Sibley DR (1990) $D_{1}$ and $D_{2}$ dopamine receptor-regulated gene expression of striatonigral and striatopallidal neurons. Science 250:14291432.

Hammer RP, Pires WS, Markou A, Koob GF (1993) Withdrawal following cocaine self-administration decreases regional cerebral metabolic rate in critical brain reward regions. Synapse 14:73-80.

Heimer L, Zahm DS, Churchill L, Kalivas PW, Wohltman C (1991) Specificity in the projection patterns of accumbers core and shell in the rat. Neuroscience 41:89-125.

Henry DJ, White FJ (1991) Repeated cocaine administration causes persistent enhancement of $\mathrm{D}_{1}$ dopamine receptor sensitivity within the rat nucleus accumbens. J Pharmacol Exp Ther 258:882-889.

Henry DJ, Greene MA, White FJ (1989) Electrophysiological effects of cocaine in the mesoaccumbens dopamine system-repeated administration. J Pharmacol Exp Ther 251:833-839.

Herkenham M, Nauta WJH (1977) Afferent connections of the habenular nuclei in the rat. A horseradish peroxidase study with a note on the 'fiber-of-passage' problem. J Comp Neurol 173:123-146. 
Hope B, Kosofsky B, Hyman SE, Nestler EJ (1992) Regulation of immediate early gene expression and AP-1 binding in the rat nucleus accumbens by chronic cocaine. Proc Natl Acad Sci USA 89:57645768.

Hurd YL, Weiss E, Koob GF, Anden N-E, Ungerstedt U (1989) Cocaine reinforcement and extracellular dopamine overflow in rat nucleus accumbens: an in vivo microdialysis study. Brain Res 498:199203.

Imperato A, Mele A, Scrocco MG, Puglisi AS (1992) Chronic cocaine alters limbic extracellular dopamine. Neurochemical basis for addiction. Eur J Pharmacol 212:299-300.

Izenwasser S, Cox BM (1992) Inhibition of dopamine uptake by cocaine and nicotine: tolerance to chronic treatments. Brain Res 573: $119-125$.

Javaid JI, Davis JM (1993) Cocaine disposition in discrete regions of rat brain. Biopharm Drug Disp 14:357-364.

Kadekaro M, Vance WH, Terrell ML, Gary JH, Eisenberg HM, Sokoloff $\mathrm{L}$ (1987) Effects on antidromic stimulation of the ventral root on glucose utilization in the ventral horn of the spinal cord in the rat. Proc Natl Acad Sci USA 84:5492-5495.

Kalivas PW, Duffy P (1993) Time course of extracellular dopamine and behavioral sensitization to cocaine. I. Dopamine axon terminals. J Neurosci 13:266-275.

Kalivas PW, Duffy P, DuMars LA, Skinner C (1988) Behavioral and neurochemical effects of acute and daily cocaine administration in rats. J Pharmacol Exp Ther 245:485-492.

Kawaguchi Y, Wilson CJ, Emson PC (1990) Projection subtypes of rat neostriatal matrix cells revealed by intracellular injection of biocytin. J Neurosci 10:3421-3438.

King GR, Joyner C, Lee TH, Kuhn C, Ellinwood EH (1992) Intermittent and continuous cocaine administration: residual behavior states during withdrawal. Pharmacol Biochem Behav 43:243-248.

Kleven MS, Perry BD, Woolverton WL, Seiden LS (1990) Effects of repeated injection of cocaine on $D_{1}$ and $D_{2}$ dopamine receptors in rat brain. Brain Res 532:265-270.

Kornetsky C, Huston-Lyons D, Porrino LJ (1991) The role of the olfactory tubercle in the effects of cocaine, morphine and brain-stimulation reward. Brain Res 541:75-81.

London ED, Wilkerson G, Goldberg SR, Risner ME (1986) Effects of $\mathrm{L}$-cocaine on local cerebral glucose utilization in the rat. Neurosci Lett 68:73-78.

London ED, Cascella NG, Wong DF, Phillips RL, Dannals RF, Links JM, Herning R, Grayson R, Jaffe JH, Wagner HN (1990a) Cocaineinduced reduction of glucose utilization in human brain - a study using positron emission tomography and [fluorine-18]-fluorodeoxyglucose. Arch Gen Psychiatry 47:567-574.

London ED, Wilkerson G, Ori C, Kimes AS (1990b) Central action of psychomotor stimulants on glucose utilization in extrapyramidal motor areas of the rat brain. Brain Res 512:155-158.

Mayfield RD, Larson G, Zahniser NR (1992) Cocaine-induced behavioral sensitization and $D_{1}$ dopamine receptor function in rat nucleus accumbens and striatum. Brain Res 573:331-335.

McCulloch J, Savaki HE, McCulloch MC, Jehle J, Sokoloff L (1982) The distribution of alterations in energy metabolism in the rat brain produced by apomorphine. Brain Res 243:67-80.

Moratalla R, Graybiel AM, Robertson HA (1992) Dynamic regulation of NGFI-A (zif $268, e g r 1)$ gene cxpression in the striatum. J Ncurosei 12:2609-2622

Moratalla R, Vickers EA, Robertson HA, Cochran BH, Graybiel AM (1993) Coordinate expression of $\mathrm{c}-f o s$ and jun $\mathrm{B}$ is induced in the rat striatum by cocaine. J Neurosci 13:423-433.

Pan HT, Menacherry S, Justice JB (1991) Differences in the pharmacokinetics of cocaine in naive and cocaine-experienced rats. J Neurochem 56:1299-1306.

Paxinos $\mathrm{G}$, Watson C (1986) The rat brain in stereotaxic coordinates. New York: Academic.

Pearlson GD, Jeffery PJ, Harris GJ, Ross CA, Fischman MW, Camargo EE (1993) Correlation of acute cocaine-induced changes in local cerebral blood flow with subjective effects. Am J Psychiatry 150:495497.

Peris J, Boyson SA, Cass WA, Curella P, Dwoskin LP, Larson G, Lin L-H, Uasuda RP, Zahniser NR (1990) Persistence of neurochemical changes in dopamine systems after repeated cocaine administration. J Pharmacol Exp Ther 253:38-44.

Pettit HO, Justice JB (1989) Dopamine in the nucleus accumbens during cocaine self-administration as studied by in vivo microdialysis. Pharmacol Biochem Behav 34:899-904.

Pettit HO, Pan H-T, Parsons LH, Justice JB (1990) Extracellular concentrations of cocaine and dopamine are enhanced during chronic cocaine administration. J Neurochem 55:798-804.

Porrino LJ, Domer FR, Crane AM, Sokoloff L (1988) Selective alterations in cercbral metabolism within the mesocorticolimbic dopaminergic system produced by acute cocaine administration in rats. Neuropsychopharmacology 1:109-1 18 .

Post RM, Weiss SRB, Smith JJ, Sullivan TL, Pert A (1987) Amygdala and nucleus accumbens lesions impair cocaine sensitization. Soc Neurosci Abstr 13:661.

Reith ME, Benuck M, Lajtha A (1987) Cocaine disposition in the brain after continuous or intermittent treatment and locomotor stimulation in mice. J Pharmacol Exp Ther 243:281-287.

Ritz MC, Lamb R J, Goldberg SR, Kuhar MJ (1987) Cocaine receptors on dopamine transporters are related to self-administration of cocaine. Science 237:1219-1223.

Roberts DCS, Koob GF, Klonoff P, Fibiger HC (1980) Extinction and recovery of cocaine self-administration following 6-hydroxydopamine lesions of the nuclcus accumbens. Pharmacol Biochem Bchav 12: $781-787$.

Robledo P, Koob GF (1993) Two discrete nucleus accumbens projection areas differentially mediate cocaine self-administration in the rat. Behav Brain Res 55:159-166.

Schwartz WJ, Smith CB, Davidsen L, Savaki H, Sokoloff L, Mata M, Fink DJ, Gainer H (1979) Metabolic mapping of functional activity in the hypothalamoneurohypophyseal system of the rat. Science 205 : 723-725.

Segal DS, Kuczenski R (1992) Repeated cocaine administration induces behavioral sensitization and corresponding decreased extracellular dopamine responses in caudate and accumbens. Brain Res 577:351-355.

Sharkey J, McBean DE, Kelly PAT (1991) Acute cocaine administration: cffects on local cerebral blood flow and metabolic demand in the rat. Brain Res 548:310-314.

Sharp FR, Gonzalez MF, Sharp JW, Sagar SM (1989) c-fos Expression and $\left({ }^{14} \mathrm{C}\right) 2$-deoxyglucose uptake in the caudal cerebellum of the rat during motor/sensory cortex stimulation. J Comp Neurol 284:621636.

Sharpe LG, Pilotte NS, Mitchell WM, De Souza EB (1991) Withdrawal of repeated cocaine decreases autoradiographic $\left[{ }^{3} \mathrm{H}\right]$ mazindol-labelling of dopamine transporter in rat nucleus accumbens. Eur J Pharmacol 203:141-144.

Skagerberg G, Lindvall O, Bjorklund A (1984) Origin, course and termination of the mesohabenular dopamine pathway in the rat. Brain Res 307:99-108.

Sokoloff L, Reivich M, Kennedy C, Des Rosiers MH, Patlak CS, Pettigrcw KD, Sakurada O, Shinohara M (1977) The $\left[{ }^{14} \mathrm{C}\right]$ deoxyglucose method for the measurement of local cerebral glucose utilization: theory, procedure, and normal values in the conscious and anesthetized albino rat. J Neurochem 28:897-916.

Terwilliger RZ, Beitner-Johnson D, Sevarino KA, Crain SM, Nestler EJ (1991) A general role for adaptations in G-proteins and the cyclic AMP system in mediating the chronic actions of morphine and cocaine on neuronal function. Brain Res 548:100-110.

Trugman JM, James CL (1993) $D_{1}$ dopamine agonist and antagonist effects on regional cerebral glucose utilization in rats with intact dopaminergic innervation. Brain Res 607:270-274.

van der Kooy D, Carter DA (1981) The organization of the efferent projections and striatal afferents of the entopeduncular nucleus and adjacent areas in the rat. Brain Res 211:15-36.

Volkow ND, Hitzemann R, Wang G-J, Fowler JS, Wolf AP, Dewey S, Handlesman $L$ (1992) Long-term frontal brain metabolic changes in cocaine abusers. Synapse 11:184-190.

Weiss F, Paulus MP, Lorang MT, Koob GF (1992) Increases in extracellular dopamine in the nucleus accumbens by cocaine are inversely related to basal levels: effects of acute and repeated administration. J Neurosci 12:4372-4380.

Weiss SRB, Post RM, Pert A, Woodward R, Murman D (1989) Context-dependent cocaine sensitization: differential effect of haloperidol on development versus expression. Pharmacol Biochem Behav 34 655-661.

West MO, Peoples LL, Wolske M, Dworkin SI (1992) Psychomotor stimulant effects on single neurons in awake, behaving rats (Frascella J, Brown RM, eds), pp 57-71. Washington: DHHS. 
Woolverton WL, Kleven MS (1988) Evidence for cocaine dependence in monkeys following a prolonged period of exposure. Psychopharmacology (Berl) 94:288-291.

Young ST, Porrino LJ, Iadarola MJ (1991) Cocaine induces striatal c-fos-immunoreactive proteins via dopaminergic $\mathrm{D}_{1}$ receptors. Proc Natl Acad Sci USA 88:1291-1295.
Zahm DS, Brog JS (1992) On the significance of subterritories in the "accumbens" part of the rat ventral striatum. Neuroscience 50:751767. 